\title{
Generalized Wave Energy Expressions in Strongly Dispersive Media in Terms of Bilinear Concomitants
}

\author{
D. ANDERSON \\ Research Laboratory of Electronics, Chalmers University of Technology, Gothenburg, Sweden \\ (Z. Naturforsch. 27 a, 1571-1576 [1972] ; received 2 September 1972)
}

The standard expressions for averaged energy density and energy flow of waves in dispersive media are generalized in order to include effects due to strong dispersion and/or broad band signals. A close relationship is revealed between these generalized energy expressions and the bilinear concomitants corresponding to the dispersion operator, which governs the wave motion.

\section{Introduction}

In a previous paper ${ }^{1}$, generalized expressions were given for the averaged energy density of slowly varying electromagnetic waves in a temporally dispersive medium. The result was a generalization of the standard expression and included correction terms, which should be important for broad band signals and/or strong dispersion. It was furthermore suggested that the concept of bilinear concomitants should prove valuable in studies of generalized wave energy expressions in dispersive media.

In the present paper we will shown that this is indeed the case, because averaged energy density and energy flow turn out to be essentially the bilinear concomitants of the fundamental dispersion operator, which governs the wave motion. The obtained generalized wave energy expressions include higher order derivatives of the envelope function of the wave as well as of the characteristic dispersion function and should consequently be important for strong dispersion and/or broad band signals. The conventional expressions for averaged energy density and energy flow are obtained in the limit of weak dispersion and narrow signals. The analysis is quite general and should be applicable to all kinds of wave motion.

Finally we want to point out the important role played in the derivation by a Lagrangian density containing higher order derivatives. Lagrangians of this unusual form have recently attracted considerable theoretical interest within the domain of the so-called generalized mechanics, see ${ }^{2}$ and references therein. Parts of the results given in the present paper have been published in a preliminary letter ${ }^{3}$.

Reprint requests to D. ANDERson, Research Laboratory of Electronics, Chalmers University of Technology, S-402 20 Göteborg 5, Sweden.

\section{A Short Review}

We assume a linear, homogeneous, lossless, and dispersive wave problem characterized by the following matrix equation:

$$
\mathbf{A}\left(\frac{\partial}{\partial t}, \frac{\partial}{\partial x}\right) \mathbf{E}(t, x)=\boldsymbol{J}(t, x)
$$

$x$ and $t$ denote space and time coordinates, $\mathbf{A}$ is a linear matrix operator, and $\boldsymbol{E}$ and $\boldsymbol{J}$ are vectors specifying the wave fields and the external driving fields respectively. If we have one external field only, a "current" density $J(t, x)$, we can Fourier transform Eq. (2.1) into the scalar form, see ${ }^{4}$

$$
D(\omega, k) E(\omega, k)=i J(\omega, k)
$$

where $D(\omega, k)$ is the dispersion function, $E(\omega, k)$ and $J(\omega, k)$ are the transforms of a fundamental wave field $E(t, x)$ and the current density $J(t, x)$ respectively. $E(t, x)$ is chosen such that $E(t, x) J(t, x)$ constitutes the power density $P(t, x)$ supplied by the external source $J(t, x)$. For a full account of these matters see ${ }^{4}$. Notations and terminology are chosen with a possible application to electromagnetic waves in mind. However, this is not essential to the analysis and e.g. in a mechanical wave problem the corresponding quantities would have been the velocity field and the force density.

We shall use complex notations and consequently write the real quantity $X(t, x) \quad(X=E, J)$ as

$$
X(t, x)=\operatorname{Re}\left[X_{0}(t, x) \exp \left\{i\left(k_{0} x-\omega_{0} t\right)\right\}\right] .
$$

The fields are assumed to be slowly varying, i. e. they contain a rapidly oscillating part determined by the exponential and a slowly varying envelope function. A formal definition is given by the conditions

$$
\begin{aligned}
& \frac{\partial}{\partial t} \ln \left|X_{0}(t, x)\right| \\
& \frac{\partial}{\partial x} \ln \left|X_{0}(t, x)\right| \ll \omega_{0}, \\
& \ll k_{0} .
\end{aligned}
$$


In the case when the envelope function $X_{0}(t, x)$ is constant, i. e. the monochromatic case, it is shown in ${ }^{4}$ how Eq. (2.2) leads to an averaged energy conservation equation of the form

where

$$
\partial / \partial t\langle W\rangle+\partial / \partial x\langle S\rangle=\langle P\rangle
$$

$$
\begin{aligned}
\langle W\rangle & =\left.\frac{1}{4} \frac{\partial D(\omega, k)}{\partial \omega}\right|_{\left(\omega_{0}, k_{0}\right)} E_{0} E_{0}{ }^{*}, \\
\langle S\rangle & =-\left.\frac{1}{4} \frac{\partial D(\omega, k)}{\partial k}\right|_{\left(\omega_{0}, k_{0}\right)} E_{0} E_{0}{ }^{*}, \\
\langle P\rangle & =\frac{1}{2} \operatorname{Re}\left(E_{0}{ }^{*} J_{0}\right) .
\end{aligned}
$$

Here ${ }^{*}$ denotes complex conjugate and the physical meanings of the quantities appearing in Eq. (2.6) are: $\langle W\rangle$ - averaged energy density, $\langle S\rangle$ - averaged energy flow, and $\langle P\rangle$ - averaged power density.

The averaged Lagrangian corresponding to the free problem $(J=0)$ we denote $\widetilde{L}$ for reasons which will become clear later on. In the monochromatic case $\widetilde{L}$ is related to the dispersion function through the relation

$$
\omega \widetilde{L}=D(\omega, k) E_{0} E_{0}^{*} .
$$

For convenience we also introduce the function $\widetilde{L}^{\prime}$ given by $\widetilde{L}^{\prime}=\omega \widetilde{L}$. Then we have the results

(i) $\widetilde{L}$ gives as variational equation the dispersion relation, i. e.

$$
\left.\frac{\partial \tilde{L}}{\partial E_{0}^{*}}\right|_{\left(\omega_{0}, k_{0}\right)}=0 \Rightarrow D\left(\omega_{0}, k_{0}\right)=0 .
$$

(ii) Averaged energy density and energy flow can be expressed in terms of the averaged Lagrangian $\widetilde{L}$ (or the function $\widetilde{L^{\prime}}$ ) as follows

$$
\begin{aligned}
& \langle W\rangle=\left.\frac{1}{4} \frac{\partial(\omega \widetilde{L})}{\partial \omega}\right|_{\left(\omega_{0}, k_{0}\right)}=\left.\frac{1}{4} \frac{\partial \widetilde{L^{\prime}}}{\partial \omega}\right|_{\left(\omega_{0}, k_{0}\right)}, \\
& \langle S\rangle=-\left.\frac{1}{4} \frac{\partial(\omega \widetilde{L})}{\partial k}\right|_{\left(\omega_{0}, k_{0}\right)}=-\left.\frac{1}{4} \frac{\partial \widetilde{L^{\prime}}}{\partial k}\right|_{\left(\omega_{0}, k_{0}\right)} \mid .
\end{aligned}
$$

The aim of the present investigation is to extend Eqs. (2.6) and (2.7) for the case of a slowly varying envelope function, while preserving the form of Equation (2.9). This condition will subsequently be shown to lead to a high degree of symmetry in $\widetilde{L^{\prime}}$. Other facts supporting this approach are given in ${ }^{3}$.

\section{The Dispersion Operator}

Following the technique of paper $^{1}$ we expand the dispersion function $D(\omega, k)$ of Eq. (2.2) in Taylor series around the point $\left(\omega_{0}, k_{0}\right)$. If we multiply this equation with $\exp \left\{i\left[\left(k-k_{0}\right) x-\left(\omega-\omega_{0}\right) t\right]\right\}$ and integrate with respect to $\omega$ and $k$, it is possible to interpret powers of $\left(k-k_{0}\right)$ and $\left(\omega-\omega_{0}\right)$ as space and time derivatives respectively and we obtain an equivalent form of Eq. (2.2), viz.

$$
D_{\mathrm{op}}\left(\frac{\partial}{\partial t}, \frac{\partial}{\partial x}\right) E_{0}(t, x)=i J_{0}(t, x)
$$

where the dispersion operator $D_{\mathrm{op}}$ is given by

$$
D_{\mathrm{op}}=\sum_{n=0}^{\infty} \frac{D(\omega, k)}{i^{n} n !}\left[\frac{\overleftarrow{\partial}}{\partial k} \frac{\vec{\partial}}{\partial x}-\frac{\overleftarrow{\partial}}{\partial \omega} \vec{\partial}\right]^{n}
$$

and $D(\omega, k) \cdot \partial \overleftarrow{\partial} k$ denotes $\partial D / \partial k$ evaluated at the point $\left(\omega_{0}, k_{0}\right)$ etc.

For a lossless system $D(\omega, k)$ is real ${ }^{4}$, which implies that $D_{\text {op }}$ is a selfadjoint operator, i. e.

$$
D_{\mathrm{op}}^{*}=\overline{D_{\mathrm{op}}}
$$

where $\overline{D_{\mathrm{op}}}$ denotes the operator algebraically adjoint to $D_{\text {op }}$.

\section{The Conservation Equation}

Consider the expression $E_{0}{ }^{*} D_{\mathrm{op}} E_{0}-E_{0} \overline{D_{\mathrm{op}}} E_{0}{ }^{*}$, which can be rewritten in two ways as follows

(i) by means of Eqs. (2.2) and (3.3) we get

$$
E_{0}{ }^{*} D_{\mathrm{op}} E_{0}-E_{0} \overline{D_{\mathrm{op}}} E_{0}{ }^{*}=2 i \operatorname{Re}\left(E_{0}{ }^{*} J_{0}\right) \text {. }
$$

(ii) By means of the Lagrange identity, $\mathrm{cf}^{5}{ }^{5}$

$$
\begin{aligned}
E_{0}{ }^{*} D_{\mathrm{op}} E_{0}-E_{0} \overline{D_{\mathrm{op}}} E_{0}{ }^{*} & =\frac{\partial}{\partial t}\left[P\left(E_{0}, E_{0}{ }^{*}\right)\right] \\
& +\frac{\partial}{\partial x}\left[Q\left(E_{0}, E_{0}{ }^{*}\right)\right]
\end{aligned}
$$

where $P\left(E_{0}, E_{0}{ }^{*}\right)$ and $Q\left(E_{0}, E_{0}{ }^{*}\right)$ are the bilinear concomitants corresponding to the dispersion operator $D_{\mathrm{op}}$. In the case of a selfadjoint operator we can write

$$
\begin{aligned}
& P\left(E_{0}, E_{0}{ }^{*}\right)=i \widetilde{P}\left(E_{0}, E_{0}{ }^{*}\right), \\
& Q\left(E_{0}, E_{0}{ }^{*}\right)=i \widetilde{Q}\left(E_{0}, E_{0}{ }^{*}\right)
\end{aligned}
$$

where $\widetilde{P}\left(E_{0}, E_{0}{ }^{*}\right)$ and $\widetilde{Q}\left(E_{0}, E_{0}{ }^{*}\right)$ are real.

From Eqs. (4.1) and (4.2) we obtain

$\frac{\partial}{\partial t}\left[\widetilde{P}\left(E_{0}, E_{0}{ }^{*}\right)\right]+\frac{\partial}{\partial x}\left[\widetilde{Q}\left(E_{0}, E_{0}{ }^{*}\right)\right]=2 \operatorname{Re}\left(E_{0}{ }^{*} J_{0}\right)$.

But $\frac{1}{2} \operatorname{Re}\left(E_{0}{ }^{*} J_{0}\right)$ is equal to the averaged power density $\langle P\rangle$, and a comparison with the conserva- 
tion Eq. (2.5) yields the following identifications

$$
\begin{aligned}
\langle W\rangle & =\frac{1}{4} \widetilde{P}\left(E_{0}, E_{0}{ }^{*}\right), \\
\langle S\rangle & =\frac{1}{4} \widetilde{Q}\left(E_{0}, E_{0}{ }^{*}\right) .
\end{aligned}
$$

Thus we have shown, how the concepts of adjoint operator and bilinear concomitants come in very naturally and makes the derivation of the generalized energy expressions Eq. (4.5) formally very short. The result is appealingly simple, as averaged energy density and energy flow are (apart from the factor $i / 4$ ) the concomitants of the dispersion operator.

\section{Symmetric Concomitants}

At this stage a difficulty arises, because the Lagrange identity, Eq. (4.2), does not uniquely determine the concomitants corresponding to an operator containing mixed derivatives. This depends essentially on the fact that a mixed derivative can be split in two ways, either of which will give different contributions to the concomitants. For example the operator $\partial^{2} / \partial t \partial x$ can be written either as $\partial / \partial t(\partial / \partial x)$ or as $\partial / \partial x(\partial / \partial t)$. Consequently we need additional information in order to make a unique choice of concomitants. This can be accomplished by postulating that there is a common function $L^{\prime}=\omega L$ depending upon $D(\omega, k), E_{0}, E_{0}{ }^{*}$, and various derivatives of these quantities, such that; cf. Eqs. (2.9), (4.3), and (4.5). (We furthermore define $X=i \widetilde{X}$, where $X=L, L^{\prime}$.)

$$
\begin{aligned}
& P\left(E_{0}, E_{0}^{*}\right)=\left.\frac{\partial(\omega L)}{\partial \omega}\right|_{\left(\omega_{0}, k_{0}\right)}=\left.\frac{\partial L^{\prime}}{\partial \omega}\right|_{\left(\omega_{0}, k_{0}\right)}, \\
& Q\left(E_{0}, E_{0}^{*}\right)=-\left.\frac{\partial(\omega L)}{\partial k}\right|_{\left(\omega_{0}, k_{0}\right)}=-\left.\frac{\partial L^{\prime}}{\partial k}\right|_{\left(\omega_{0}, k_{0}\right)} .
\end{aligned}
$$

We emphasize, that although Eq. (5.1) is chosen so as to preserve the form of Eq. (2.9), it is not evident a priori that $L$ is a Lagrangian density, i. e. it will have to be proved that the variational equation of $L$ yields the correct differential equation for $E_{0}$, Equation (3.1).

Then it can be shown (see Appendix) that Eq. (6.3) termination of $l_{n, m}$, viz.

$$
(m+1) \frac{\partial l_{n, m}}{\partial t}+(n-m) \frac{\partial l_{n, m+1}}{\partial x}=E_{0}{ }^{*} D_{n+1, m+1} E_{0}+(-1)^{n} E_{0} D_{n+1, m+1} E_{0}{ }^{*} .
$$

In order to solve this equation we make the following ansatz for the functions $l_{n, m}$

$$
l_{n, m}=\sum_{\nu=0}^{m} \sum_{\mu=0}^{n-m} \lambda_{v, \mu}^{n, m}\left(\Lambda_{v, \mu}^{*} \Lambda_{m-v, n-m-\mu}+(-1)^{n} \Lambda_{\nu, \mu} \Lambda_{m-v, n-m-\mu}^{*}\right)
$$


where

$$
\Lambda_{v, \mu}=D_{v+\mu, \nu} E_{0}=\partial^{v+\mu} E_{0} / \partial t^{\nu} \partial x^{\mu} .
$$

$\lambda_{v, \mu}^{n, m}$ are coefficients to be determined and some of the anticipated symmetry in $L^{\prime}$ has been used in the ansatz.

A more convenient form of Eq. (6.8) can be obtained by a suitable change of summation indices in the last term, which gives

where

$$
l_{n, m}=\sum_{v=0}^{m} \sum_{\mu=0}^{n-m} \chi_{v, \mu}^{n, m} \Lambda_{v, \mu}^{*} \Lambda_{m-v, n-m-\mu}
$$

$$
\chi_{v, \mu}^{n, m}=\lambda_{v, \mu}^{n, m}+(-1)^{n} \lambda_{m-v, n-m-\mu}^{n, m} .
$$

From Eq. (6.11) we get the symmetry condition

$$
\chi_{v, \mu}^{n, m}=(-1)^{n} \chi_{m-v, n-m-\mu}^{n, m}
$$

which will be used later on to facilitate the solution of Equation (6.7).

Finally, insertion of the ansatz Eq. (6.10) into Eq. (6.7) yields a recurrence formula for $\chi_{v, \mu}^{n, m}$, which is solved in appendix with the result

$$
\begin{aligned}
\chi_{v, \mu}^{n, m}=(-1)^{v+\mu} & \frac{m !(n-m) !}{(n+1) !} \frac{(v+\mu) !}{v ! \mu !} \\
& \cdot \frac{(n-v-\mu) !}{(m-v) !(n-m-\mu) !} .
\end{aligned}
$$

\section{The Variational Equation}

There remains to prove that $L$ really is a Lagrangian density corresponding to the free problem $\left(J_{0}(t, x)=0\right)$, i. e. that the variational equation for $L$ gives the equation

We have

$$
D_{\text {op }} E_{0}=0 .
$$

$L^{\prime}=\sum_{n=0}^{\infty} \sum_{m=0}^{n} i A_{n, m} l_{n, m}$,

$l_{n, m}=\sum_{\nu=0}^{m} \sum_{\mu=0}^{n-m} \chi_{\nu, \mu}^{n, m} \Lambda_{\nu, \mu}^{*} \Lambda_{m-v, n-m-\mu}$.

The variational derivative of $L$ with respect to $E_{0}^{*}=\Lambda_{0,0}^{*}$ is, according to generalized mechanics

$$
\frac{\delta L}{\delta \Lambda_{0,0}^{*}}=\sum_{r=0}^{\infty} \sum_{s=0}^{\infty}(-1)^{r+s} \frac{\partial r+s}{\partial t^{r} \partial x^{s}}\left(\frac{\delta L}{\delta \Lambda_{r, s}^{*}}\right) .
$$

Inserting the expression for $L^{\prime}$, Eq. (7.2), gives

$$
\omega \frac{\delta L}{\delta \Lambda_{0,0}^{*}}=\sum_{r=0}^{\infty} \sum_{s=0}^{\infty} \sum_{n=0}^{\infty} \sum_{m=0}^{n} i A_{n, m}(-1)^{r+s} \sum_{\nu=0}^{m} \sum_{\mu=0}^{n-m} \chi_{v, \mu}^{n, m} \frac{\partial r+s}{\partial t^{r} \partial x^{s}}\left(\Lambda_{m-\nu, n-m-\mu}\right) \delta_{r \nu} \delta_{s,}
$$

where $\delta_{r v}$ is the usual Kronecker symbol. Equation (7.4) simplifies to

$$
\omega \frac{\delta L}{\delta \Lambda_{0,0}^{*-}}=\sum_{n=0}^{\infty} \sum_{m=0}^{n} i A_{n, m} \Lambda_{m, n-m} \sum_{\nu=0}^{m} \sum_{\mu=0}^{n-m}(-1)^{\nu+\mu} \chi_{\nu, \mu}^{n, m} .
$$

But the following simple relation is valid, viz.

$$
\sum_{\nu=0}^{m} \sum_{\mu=0}^{n-m}(-1)^{\nu+\mu} \chi_{v, \mu}^{n, m}=1 \text {. }
$$

This is easily proved once the $\chi_{v, \mu}^{n, m}$ 's are recognized to be essentially the coefficients of the hypergeometric probability distribution.

Using this fact Eq. (7.5) reads

$$
\omega \frac{\delta L}{\delta \Lambda_{0,0}^{*}}=\sum_{n=0}^{\infty} \sum_{m=0}^{n} i A_{n, m} \Lambda_{m, n-m}
$$

Finally setting the variational derivative equal to zero yields

$$
\left.\omega \frac{\delta L}{\delta \Lambda_{0,0}^{*}}\right|_{\left(\omega_{0}, k_{0}\right)}=i D_{\mathrm{op}} E_{0}=0
$$

and we obtain Eq. (7.1) as we should.

\section{Conclusions}

Summarizing, we have found that, (i) averaged energy density and energy flow can be expressed in terms of the bilinear concomitants of the dispersion operator [Eq. (4.5)], (ii) the concomitants are made symmetric by means of a common function $L^{\prime}$, as a consequence of the condition that the standard expression Eq. (2.9) should be form invariant, (iii) from Eqs. (4.5) and (5.1) we obtain the generalized energy expressions

$$
\begin{aligned}
\langle W\rangle & =\frac{1}{4} \widetilde{P}\left(E_{0}, E_{0}{ }^{*}\right)=\left.\frac{1}{4} \partial \tilde{L}^{\prime}\right|_{\left(\omega_{0}, k_{0}\right)}, \\
\langle S\rangle & =\frac{1}{4} \widetilde{Q}\left(E_{0}, E_{0}{ }^{*}\right)=-\frac{1}{4} \partial \tilde{L}^{\prime}{ }_{\left(\omega_{0}, k_{0}\right)} .
\end{aligned}
$$

These are the main results of our analysis and previously stated in ${ }^{3}$, however not in terms of con- 
comitants. Retaining only the first correction terms yields expressions which should be sufficient for most practical purposes. We obtain, cf. Eq. (2.6)

$$
\begin{aligned}
\langle W\rangle & =\frac{1}{4}\left[\frac{\partial D}{\partial \omega} E_{0} E_{0}^{*}-\frac{i}{2} \frac{\partial^{2} D}{\partial \omega^{2}}\left(E_{0}^{*} \frac{\partial E_{0}}{\partial t}-E_{0} \frac{\partial E_{0}{ }^{*}}{\partial t}\right)+\frac{i}{2} \frac{\partial^{2} D}{\partial \omega \partial k}\left(E_{0}^{*} \frac{\partial E_{0}}{\partial x}-E_{0} \frac{\partial E_{0}{ }^{*}}{\partial x}\right)\right]_{\left(\omega_{0}, k_{0}\right)}, \\
\langle S\rangle & =-\frac{1}{4}\left[\frac{\partial D}{\partial k} E_{0} E_{0}{ }^{*}+\frac{i}{2} \frac{\partial^{2} D}{\partial k^{2}}\left(E_{0}^{*} \frac{\partial E_{0}}{\partial x}-E_{0} \frac{\partial E_{0}^{*}}{\partial x}\right)-\frac{i}{2} \frac{\partial^{2} D}{\partial \omega \partial}\left(E_{0}^{*} \frac{\partial E_{0}}{\partial t}-E_{0} \frac{\partial E_{0}{ }^{*}}{\partial t}\right)\right]_{\left(\omega_{0}, k_{0}\right)} .
\end{aligned}
$$

These relations should be important when dispersion is strong and/or the variation of the envelope function $E_{0}$ can not be neglected. (iv) $L$ gives the correct variational equation and thus constitutes an averaged Lagrangian density for the problem, (v) Finally we note the possibility to define an energy velocity $v_{\mathrm{E}}$ by means of $\mathrm{Eq}$. (8.2) and the relation

$$
v_{\mathrm{E}}=\langle W\rangle /\langle S\rangle \text {. }
$$

This obviously leads to a velocity depending upon the form of the signal.

\section{Acknowledgement}

Part of the results presented here have been given in a joint letter ${ }^{3}$ with Dr. J. Askne. The author is greatly indebted to Dr. AsKNE for his constant interest also in the present work and for many valuable suggestions.

\section{Appendix A}

\section{Derivation of the Recurrence Formula, Eq. (6.7)}

We begin by eliminating the coefficients $A_{n, m}^{0}$ appearing in Equation (6.3). For this purpose we need the following relations, which are easily obtained from Equation (6.2).

$$
A_{n, m}=\frac{i}{m} \frac{\partial}{\partial \omega}\left(A_{n-1, m-1}\right)=-\frac{i}{n-m} \frac{\partial}{\partial k}\left(A_{n-1, m}\right)
$$

Put $X_{n, m}=A_{n, m}^{0} x_{n, m}$ where $X_{n, m}=P_{n, m}, Q_{n, m}$. Then Eqs. (6.5) and (A.1) yield

$$
\begin{aligned}
& P_{n, m}=A_{n, m}^{0} p_{n, m}=\left.\frac{i}{m} \frac{\partial}{\partial \omega}\left(A_{n-1, m-1}\right)\right|_{\left(\omega_{0}, k_{0}\right)} p_{n, m}=\left.\frac{\partial}{\partial \omega}\left(L_{n-1, m-1}\right)\right|_{\left(\omega_{0}, k_{0}\right)}, \\
& Q_{n, m}=A_{n, m}^{0} \boldsymbol{q}_{n, m}=-\left.\frac{i}{n-m} \frac{\partial}{\partial k}\left(A_{n-1, m}\right)\right|_{\left(\omega_{0}, k_{0}\right)} q_{n, m}=-\left.\frac{\partial}{\partial k}\left(L_{n-1, m}\right)\right|_{\left(\omega_{0}, k_{0}\right)} .
\end{aligned}
$$

Combining Eqs. (A.1) and (A.2) we obtain

$$
L_{n-1, m-1}=\frac{i}{m} A_{n-1, m-1} p_{n, m}, \quad L_{n-1, m}=\frac{i}{n-m} A_{n-1, m} q_{n, m} .
$$

Introducing the quantities $l_{n, m}$ defined by Eq. (6.6) yields the relations

$$
m l_{n-1, m-1}=p_{n, m},(n-m) l_{n-1, m}=q_{n, m} \text {. }
$$

Divide Eq. (6.3) with $A_{n, m}^{0}$ and make use of Equation (A.4). This yields after a rising of indices the recurrence formula Equation (6.7).

\section{Appendix B}

\section{Determination of the Coefficients $\chi_{v, \mu}^{n, m}$}

Insertion of the ansatz Eq. (6.10) into the recurrence formula Eq. (6.7) gives after some rearranging in order to facilitate an identification with the right hand side of Equation (6.7)

$$
\begin{aligned}
(m+1) & \frac{\partial l_{n, m}}{\partial t}+(n-m) \frac{\partial l_{n, m}}{\partial x} \\
& \equiv(n-m)\left\langle\sum_{\nu=1}^{m} \sum_{\mu=1}^{n-m-1}\left[\chi_{v, \mu}^{n, m+1}+\chi_{v, \mu-1}^{n, m+1}+\frac{m+1}{n-m}\left(\chi_{\nu, \mu}^{n, m}+\chi_{\nu-1, \mu}^{n, m}\right)\right] \Lambda_{\nu, \mu}^{*} \Lambda_{m+1-v, n-m-\mu}\right.
\end{aligned}
$$




$$
\begin{aligned}
& +\sum_{v=1}^{m}\left\{\left[\chi_{v, n-m}^{n, m}+\frac{m+1}{n-m}\left(\chi_{v, 0}^{n, m}+\chi_{v-1,0}^{n, m}\right) \Lambda_{v, 0}^{*} \Lambda_{m+1-v, n-m}+\chi_{v, n-m-1}^{n, m}+\frac{m+1}{n-m}\left(\chi_{v, n-m}^{n, m}\right.\right.\right. \\
& \left.\left.+\chi_{\nu-1, n-m}^{n, m}\right) \Lambda_{v, n-m}^{*} \Lambda_{m+1-v, 0}\right\}+\sum_{\mu=1}^{n-m-1}\left\{\left[\left(\chi_{0, \mu}^{n, m-1}+\chi_{0, \mu-1}^{n, m+1}+\frac{m+1}{n-m} \chi_{0, \mu}^{n, m}\right)\right] \Lambda_{0, \mu}^{*} \Lambda_{m+1, n-m-\mu}\right. \\
& \left.+\left(\chi_{m+1, \mu}^{n, m+1}+\chi_{m+1, \mu-1}^{n, m+1}+\frac{m+1}{n-m} \chi_{m, \mu}^{n, m}\right) \Lambda_{m+1, \mu}^{*} \Lambda_{0, n-m-\mu}\right\}+\left(\chi_{0,0}^{n, m+1}+\frac{m+1}{n-m} \chi_{0,0}^{n, m}\right) \Lambda_{0,0}^{*} \Lambda_{m+1, n-m} \\
& +\left(\chi_{m+1, n-m}^{n, m+1}+\frac{m+1}{n-m} \chi_{m, n-m-1}^{n, m}\right) \Lambda_{m+1, n-m}^{*} \Lambda_{0,0}+\left(\chi_{m+1,0}^{n, m+1}+\frac{m+1}{n-m} \chi_{m, 0}^{n, m}\right) \Lambda_{m+1,0}^{*} \Lambda_{0, n-m} \\
& \left.+\left(\chi_{0, n-m-1}^{n, m+1}+\frac{m+1}{n-m} \chi_{0, n-m}^{n, m}\right) \Lambda_{0, n-m}^{*} \Lambda_{m+1,0}\right\rangle \equiv \Lambda_{0,0}^{*} \Lambda_{m+1, n-m}+(-1)^{n} \Lambda_{n+1, n-m}^{*} \Lambda_{0,0} .
\end{aligned}
$$

Identification of the various terms gives

$$
\begin{aligned}
& (n-m)\left(\chi_{0,0}^{n, m+1}+\chi_{0,0}^{n, m}\right)=1, \\
& \chi_{m+1,0}^{n, m+1}+\frac{m+1}{n-m} \chi_{m, 0}^{n, m}=0, \\
& \chi_{v, 0}^{n, m+1}+\frac{m+1}{n-m}\left(\chi_{\nu 0}^{n, m}+\chi_{\nu-1,0}^{n, m}\right)=0, \quad 1 \leqq v \leqq m, \\
& \chi_{0, \mu}^{n, m+1}+\chi_{0, \mu-1}^{n, m+1}+\frac{m+1}{n-m} \chi_{0, \mu}^{n, m}=0, \quad 1 \leqq \mu \leqq n-m-1, \\
& \chi_{v, \mu}^{n, m+1}+\chi_{v, \mu-1}^{n, m+1}+\frac{m+1}{n-m}\left(\chi_{v, \mu}^{n, m}+\chi_{\nu-1, \mu}^{n, m}\right)=0, \quad\left\{\begin{array}{l}
1 \leqq v \leqq m, \\
1 \leqq \mu \leqq n-m-1 .
\end{array}\right.
\end{aligned}
$$

The remaining equations needed to make the identification complete are contained in Eq. (B.2) together with the symmetry condition Equation (6.12).

Several of the recurrence formulas of Eq. (B.2) are rather easy to solve, e. g. the two first readily give

$$
\chi_{0,0}^{n, m}=1 /(n+1) . \quad \chi_{m, 0}^{n, m}=(-1)^{m} \frac{m !(n-m) !}{(n+1) !} .
$$

However the last formula of Eq. (B.2), which is the most general, looks rather difficult to solve directly and we prefer to solve it by making a qualified guess at the proper appearance of the $\chi_{v, \mu}^{n, m}$ 's guided by symmetry and the simpler formulas obtained from the first relations of Equation (B.2). The solution is found to be

$$
\chi_{v, \mu}^{n, m}=(-1)^{\nu+\mu} \frac{m !(n-m) !}{(n+1) !} \frac{(v+\mu) !}{v ! \mu !} \frac{(n-v-\mu) !}{(m-v) !(n-m-\mu) !}
$$

This is easily verified by direct substitution into Equation (B.2).

The symmetry of the $\chi_{v, \mu}^{n, m}$ 's is remarkably simple. Note that they are connected with the factors, Equation $(6.10)$ :

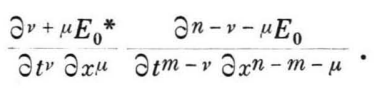

1 D. Anderson, Z. Naturforsch. 27 a, 1094 [1972].

2 M. Borneas, Amer. J. Phys. 40, 248 [1972].

3 D. Anderson and J. Askne, Proc. IEEE 60 (Letters), 901 [1972].
4 J. Askne, Int. J. Electronics 32, 573 [1972].

5 P. M. MORSE and H. Feshbach, Methods of Theoretical Physics, Vol. 1, McGraw-Hill, New York 1951. 